\title{
Jakob Balde über Epiktet und sein Encheiridion ${ }^{1}$
}

\author{
Klaus Döring \\ Otto-Friedrich-Universität Bamberg, Klassische Philologie \\ An der Universität 5, 96047 Bamberg, Deutschlad; doering.klaus@web.de
}

For citation: Klaus Döring. Jakob Balde über Epiktet und sein Encheiridion. Philologia Classica 2018, 13(1), 141-150. https://doi.org/10.21638/11701/spbu20.2018.111

The wholesale denunciation of 'Epictetus and other Stoics' found in the Odes of Jacob Balde (1604-1668), a Jesuit and a poet, comes as a surprise amid general admiration for the Encheiridion in the Christian circles and the eagerness to marry Stoicism with Christianity evident even at times when such marriage would come at a stretch. Since Epictetus offers himself for such adaptations with ease, the case of Balde requires looking into it. The disdain poured out by Balde with all probability has its causes not in his thorough — or indeed at least passing acquaintance with the Encheiridion itself, but in the anonymous compilation De vera sapientia, virtute et tranquillitate animi enchiridion DD. Eucherii Lugdunensis, Martini Bracarensis, et Magni Wigonis Antistitum of 1639, current under the name of Guigo de Castro (Wigo of Balde's Latin ode). The compiler wages a war on Stoicism providing his reader with a repository of violently, albeit unwittingly, perverted examples from the Encheiridion (the crushed seashells and earthenware pot lamp doubling as a chamber pot obviously being the go-for favourites of his sources) which Balde, who probably was not acquainted with Epictetus firsthand, only too eagerly used as a foil for his image of Paul the Apostle.

Keywords: Epictetus, Balde, Guigo de Castro, Encheiridion.

Bei den christlichen Mönchen der Spätantike und der frühen Neuzeit erfreute sich Epiktets $^{2}$ Encheiridion großer Wertschätzung. Zeugnisse dafür sind für die Spätantike die drei erhaltenen christlichen Adaptionen des Encheiridions und die erhaltenen Teile eines Kommentars zu einer dieser Adaptionen, der sog. Paraphrasis Christiana, ${ }^{3}$ für die frühe Neuzeit — der Gebrauch, den der „China-Missionar“ Matteo Ricci (1552-1610) in seinem in chinesischer Sprache verfassten moralphilosophischen Werk mit dem Titel Ershiwu yan von der Schrift machte, die Umformung des Encheiridions zu einem Enchiridion Cartusianum durch den Kartäusermönch Matthias Mittner (1575-1632), ${ }^{4}$ oder auch das emphatische Lob, mit dem Jeremias Drexel (1581-1638), Baldes Vorgänger im Amt des bayerischen Hofpredigers ${ }^{5}$ und wie dieser und Ricci Jesuit, das Encheiridion in seinem Heliotropium von

${ }^{1}$ Dieser Beitrag stellt eine Ergänzung zu dem dar, was ich in der Abhandlung „Zur Rezeption von Epiktets Encheiridion“ dargelegt habe (Döring 2010, 319-343). — Für wertvolle Hinweise danke ich Konrad Heldmann, Bernhard Herzhoff und Werner Taegert.

2 Wie üblich spreche ich vom Encheiridion und den Dissertationes so, als habe nicht Epiktets Schüler Arrian, sondern Epiktet selbst die beiden Schriften verfasst. Vgl. dazu Döring 2010, 319.

3 Die Texte der drei Adaptionen bei Boter 1999, der Text des Kommentars bei Spanneut 2007. Zu den christlichen Adaptionen des Encheiridions zuletzt Vollenweider 2013, 120-125.

${ }^{4} \mathrm{Zu}$ Riccis und Mittners Umformungen vgl. Döring 2010, 331-334.

${ }^{5}$ Vgl. Baldes Nachruf auf Drexel lyr. 1, 16.

(C) St. Petersburg State University, 2018 
1627 bedachte. Dort schreibt er: ${ }^{6}$ „Epiktet hat außer den Dissertationes auch das Encheiridion geschrieben, das man mit Zedernöl $1^{7}$ und goldenen Lettern aufzeichnen sollte. Dieses Büchlein enthält so viel gottesfürchtigen Geist und verborgene Weisheit, dass man meinen könnte, es sei von einem äußerst gottesfürchtigen Mann geschrieben worden. Dieses Büchlein wird noch am Tag des Jüngsten Gerichtes sehr viele Christen vor Scham erröten lassen, die einerseits sehr schmutzige Dinge geschrieben und andererseits kein untadeliges Leben geführt haben."

Es gab aber auch kritische Stimmen. In einer der drei Oden, in denen er sich von der stoischen Philosophie mit Entschiedenheit distanziert, der Ode 3, 39, ${ }^{8}$ spricht Balde der stoischen Philosophie im Allgemeinen und Epiktets Encheiridion im Besonderen jeden Wert ab. Die Ode trägt die Überschrift: M. P. Wigonis gravissimas considerationes de tranquillitate animi doctrina S. Pauli firmatas Epicteti aliorumque Stoicorum verbosis sententiis praefert. Diese Überschrift fasst den Inhalt der Verse 1-15 der Ode zusammen: ${ }^{9}$

\author{
Si Paulus gladium dedit, \\ Servi ridiculus quo mihi pugio? \\ Qui vultus nitidos cupis \\ Naturae vacuis discere sensibus \\ Et rebus male splendidis \\ Fallacis folium demere bracteae, \\ Wigonem lege: neu mihi \\ Occurrant solito murmure Stoici et \\ Simi cochleolas Phrygis \\ Fumantemque nigro lumine lampada, \\ Neu cassas cerebro nuces \\ Barbati pueri spargere gestiant. \\ Odi torva crepundia et \\ Pompam stultitiae nominis additam \\ Et ridenda tonitrua.

„Wenn Paulus mir ein Schwert gab, wozu soll mir dann der Dolch des Sklaven von Nutzen sein? Der du die schöne Gestalt der Natur mit unbefangenen Sinnen kennenzulernen und den in übler Weise glänzenden Dingen die Folie ihres trügerischen Blattgoldes wegzunehmen begehrst, lies Wigo. Nicht sollen mir die Stoiker in den Weg treten mit ihrem gewohnten Gemurmel von den Meeresschnecken des plattnasigen Phrygers und der von schwarzem Licht qualmenden Lampe,

${ }^{6}$ Heliotropium II 3, 2 (Drexel 1627, 106-107): Epictetus praeter dissertationes etiam Enchiridion scripsit cedro et auro exarandum. Hic libellus tantum religiosi spiritus habet et arcanae sapientiae, ut eum a viro religiosissimo putes conscriptum. Hic libellus supremi judicii die in ruborem dabit Christianorum plurimos, qui et spurcissima scripserunt nec vixerunt sanctius. Den Hinweis auf diese Stelle verdanke ich Pörnbacher 1965, 161-162, der den Text in der sehr freien deutschen Übersetzung von Joachim Meichel zitiert, die im gleichen Jahr wie die Originalausgabe (1627) veröffentlicht wurde.

7 Zedernöl wurde in der Antike benutzt, um Bücher gegen Fäulnis und Bücherwürmer zu schützen, vgl. Hor. Ars 332. Vitr. 2, 9, 13. Persius bezeichnet literarische Werke von unvergänglichem Wert als cedro digna (Sat. 1, 42). Erasmus führt diese Formel unter den antiken Sprichwörtern an (Adagia 4, 1, 54), auch Balde benutzt sie (Urania victrix 2, 5, 7, opp. poet. omn. 5, 90).

${ }^{8}$ Die beiden anderen sind 3, 12 (Überschrift: Auctor a celeberrima secta Stoicorum desciscit) und 3, 16 (Überschrift: Ad Molardum Bucconem, cur a Stoicis secesserit). Vgl. zu ihnen Arend 2006.

9 Text nach Mueller 1884. 
und nicht sollen die bärtigen Kinder ${ }^{10}$ danach trachten, taube Nüsse ${ }^{11}$ in meinem Gehirn zu verstreuen. Ich verabscheue die wilden Klappern ${ }^{12}$ und das Gepränge des Namens, der der Torheit beigelegt ist, ${ }^{13}$ und die lachhaften Donnerschläge."

Wie gegensätzlich Balde den Wert von Wigos considerationes de tranquillitate animi einerseits und Epiktets Encheiridion andererseits einschätzt, drückt sich schon rein äußerlich darin aus, wie er die beiden Autoren einführt. Im Falle Wigos nennt er nur kurz und knapp den Namen (V.7), zu seiner Person sagt er kein einziges Wort und (anders als in der Überschrift) auch nicht zu dem, was man von ihm lesen soll. Ganz anders in Fall Epiktets: Statt seinen Namen zu nennen, spricht Balde von Epiktet in offenkundig abwertender Absicht als dem Sklaven (V.2) und dem Phryger (V.9), womit er darauf anspielt, dass Epiktet als Sklave in Hierapolis in Phrygien geboren wurde. Dass Epiktet „plattnasig“ (V.9) war, ist übrigens, soweit ich sehe, nirgends bezeugt. Wenn Balde ihn so bezeichnet, ist dies vermutlich in der Weise zu erklären, dass er ihn mit diesem Attribut in die Nachfolge des plattnasigen „Urphilosophen“ Sokrates einordnet. Das Werk Epiktets, um das es geht, tituliert Balde als „den lächerlichen Dolch“ (V.2). Um dies zu verstehen, muss man Fol-

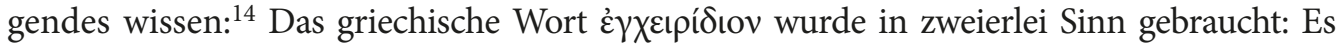
bedeutete einerseits Handwaffe = Dolch, andererseits wurde mit ihm eine Schrift bezeichnet, mit der man die Kernlehren einer bestimmten Disziplin „,in den Händen hält“, also ein Handbuch (lateinisch manuale). Diese Doppeldeutigkeit hatte sich der Neuplatoniker Simplikios in seinem Kommentar zu Epiktets Encheiridion zunutze gemacht, um den Charakter des Werkes zu beschreiben. ${ }^{15}$ In der Einleitung zu diesem Kommentar schreibt er (Hadot 1996, 196): Epiktets Büchlein „trägt den Titel Encheiridion, weil die, die gut leben wollen, es stets zur Hand und verfügbar haben sollen, wie ja auch die Handwaffe der Soldaten ein Schwert ist, welches die, die es benutzen, ständig zur Hand haben sollen." Als zu Beginn des 16. Jh. zunächst das Encheiridion und wenig später Simplikios' Kommentar dazu im Westen bekannt

${ }_{10}$ Die Stoiker, die den Philosophenbart tragen, deren Denken aber das von Kindern ist.

11 Als „taube Nuss“ bezeichneten die Römer wie wir redensartlich etwas, was sich als völlig wert- und nutzlos erweist; vgl. Plaut. Pseud. 371; Hor. Sat. 2, 5, 36.

12 Zum komplexen Bedeutungsspektrum des Wortes crepundia vgl. Leumann 1959, 193-195. Balde gebraucht das Wort hier in der Bedeutung, in der mit ihm kastagnettenartige Klappern bezeichnet wurden,

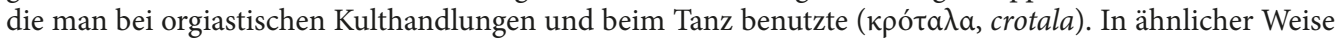
spricht der Autor des Vorwortes zu der Ausgabe von Wigos Schrift De tranquillitate animi, der Balde seine Kenntnis der Schrift verdankte (Wigo 1639), in Bezug auf die Philosophie der Stoiker von den Stoicorum crepitacula.

$13 \mathrm{Zu}$ pompam stultitiae nominis additam: In den $\$ \$ 10-11$ seiner Epistula de tribus quaestionibus behandelt Bonaventura die Frage, wem es erlaubt sein soll, als „Meister“ (magister) die Lehrkanzel zu betreten (Opp. omn., vol. 8, Quaracchi 1898, 334-335). Seine Antwort in Kurzform: Wenn Jesus zu seinen Jüngern sagt (Übers. Luther): „Aber ihr sollt euch nicht Rabbi nennen lassen; denn einer ist euer Meister; ihr aber seid alle Brüder" (Mt. 23, 8 [Text der Vulgata]: Vos autem nolite vocari rabbi, unus enim magister vester, omnes autem vos fratres estis), dann dürfe man daraus nicht herleiten, dass es generell verboten sei, als Lehrmeister aufzutreten. Allerdings dürften dies nur solche, die über das nötige Wissen verfügten, also nur Kleriker. Sein Fazit: „Ich sage also, dass gemäß der Aussage des Evangeliums Ehrgeiz und Gepränge dieses Namens (sc. des Namens magister) zu verurteilen und keineswegs zu erstreben sind, die Aufgabe aber anzunehmen ist (dico ergo, quod secundum dictum evangelicum ambitio et pompa huius nominis condemnanda est et nullatenus appetenda, sed officium assumendum). Pompa huius nominis meint hier das geltungssüchtige Prunken mit dem Titel magister. Analog dazu scheint mir mit pompa stultitiae nominis addita das geltungssüchtige Prunken mit dem Titel „Stoiker“ zu sein, das umso mehr zu verurteilen ist, als die Philosophie der Stoiker in Wirklichkeit nichts anderes als Dummheit ist.

14 Das Folgende ausführlicher in: Döring 2010, 319-320.

$15 \mathrm{Ob}$ dies auch vor ihm schon geschehen war, wissen wir nicht. 


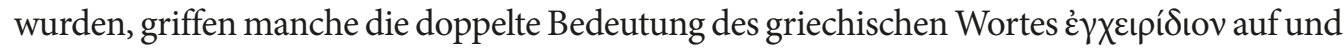
übersetzten den Titel Encheiridion entsprechend ins Deutsche und Lateinische. So veröffentlichte Jacob Schenck 1534 seine Übersetzung des Encheiridions ins Deutsche unter dem Titel Eyn schon nutzlich Büchlin genant der Sticher des Hochweysen Heiden Epicteti. Natürlich war Jacob Schenck die doppelte Bedeutung des griechischen Wortes $\dot{\varepsilon} \gamma \chi \varepsilon\llcorner\rho i ́ \delta ı$ ov bekannt. Bevor die Übersetzung beginnt, wiederholt er den Titel, diesmal in folgender Form: „Der Stechtegen oder Handtbüchlin des hochberümpten heidnischen Meisters Epicteti“. Hieronymus Wolf publizierte seine griechisch-lateinische Ausgabe des Encheiridions 1560 unter dem Titel Epicteti Enchiridion, hoc est Pugio, sive Ars humanae vitae correctrix.

Die Ode beginnt mit einem Paukenschlag: Balde stellt dem „lächerlichen Dolch des Sklaven“ das "Schwert" gegenüber, das Paulus den Menschen gegeben habe. Das ist ein Verweis auf die Aufforderung, die Paulus im Epheserbrief an die Epheser richtet (Übers. Luther): „Nehmt ... das Schwert des Geistes, welches ist das Wort Gottes“ ${ }^{16}$ Das Schwert, das Paulus den Menschen gab, ist das Wort Gottes, wie Paulus es gelehrt hat (die doctrina S. Pauli der Überschrift).

Deutliche Anspielungen auf Epiktet enthalten die Verse 9 und 10. Die „Meeresschnecken des plattnasigen Phrygers“ (V. 9) gehen auf Ench. 7 zurück; nur hier kommt das grie-

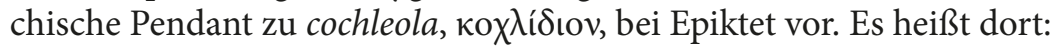

„Wenn bei einer Seereise das Schiff vor Anker gegangen ist und du aussteigst, um frisches Was-

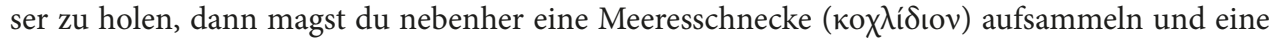
Tulpenzwiebel ( $\beta$ o $\lambda \beta a ́ p ı o v),{ }^{17}$ aber deine Aufmerksamkeit muss auf das Schiff gerichtet bleiben, und du musst dich ständig umdrehen, um zu schauen, ob nicht etwa der Kapitän ruft, und wenn er ruft, dann musst du alle jene Dinge liegen lassen, damit du nicht gefesselt wie die Schafe in das Schiff geworfen wirst. So ist es auch im Leben: Wenn dir statt einer Tulpenzwiebel und einer

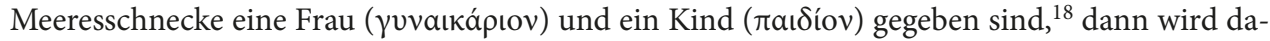
gegen nichts einzuwenden sein. Wenn aber der Kapitän (sc. Gott) ruft, dann lass alle jene Dinge liegen und lauf zum Schiff, ohne dich umzudrehen."

Man fragt sich natürlich, warum Balde gerade auf die „Meeresschnecken des plattnasigen Phrygers“ als Beispiel dafür verweist, dass es nutzlos sei, sich mit dessen philo-

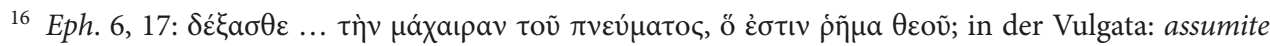
gladium spiritus quod est verbum Dei.

17 Boגßápıov wird zumeist mit „Meerzwiebel“ übersetzt. (Sicher falsch ist die Übersetzung „Tintenfischchen“, die man bisweilen liest.) Das kann nicht richtig sein. Die großen bitteren Knollen der Meerzwiebel nutzte man zu pharmazeutischen Zwecken, oder man hängte sie als Mittel zur Abwehr böser Geister über die Haustür (vgl. Hünemörder 1999). Was, so fragt man sich, soll es für einen Sinn haben, am Meeresstrand neben der eigentlichen Aufgabe, derentwegen man das Schiff verlassen hat, nämlich frisches Wasser

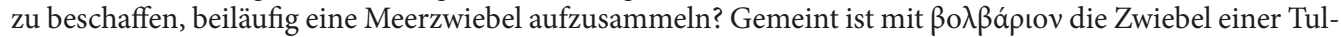
penart, die Theophrast in seiner Historia plantarum $(7,13,8)$ beschreibt (vgl. dazu Amigues 2003, 159-160; Tan u. a 2009, 146-147). Sie wächst, so Theophrast, an Meeresstränden, und das Innere ihrer Zwiebel ist essbar und schmeckt süß. Was die Person, die das Schiff verlassen hat, um Wasser zu holen, macht, ist dies, dass sie nebenbei eine Meeresschnecke und eine Zwiebel einer solchen Tulpe aufsammelt, um sie, natürlich roh, zu essen. Dass dies gemeint ist, war allerdings schon bald nicht mehr klar. Frühestes erhaltenes Zeugnis dafür ist jene christliche Adaption des Encheiridions, der man den Titel Paraphrasis Christiana gegeben hat.

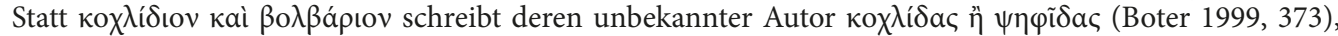
denkt also nicht an etwas, was man zum Essen, sondern etwas, was man zum Spaß sammelt.

${ }_{18}$ Meeresschnecke, Tulpenzwiebel, Frau, Kind: Epiktet gebraucht in allen vier Fällen, wie er es auch sonst mit Vorliebe tut, Deminutive, um darauf hinzuweisen, dass es sich um Dinge handelt, die letztlich

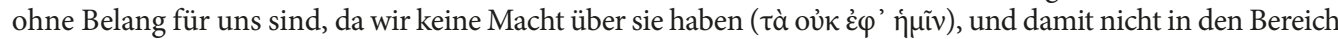

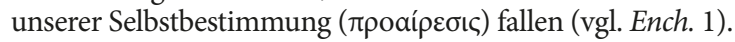


sophischen Anschauungen zu beschäftigen. Um diese Frage beantworten zu können, ist es nötig, den Blick auf die Quelle zu richten, der Balde seine Kenntnis von Wigos considerationes de tranquillitate animi verdankte. Es ist dies offenkundig der Sammelband mit dem Titel De vera sapientia, virtute et tranquillitate animi enchiridion DD. (= Dominorum) Eucherii Lugdunensis, Martini Bracarensis, et Magni Wigonis Antistitum, der 1639, also vier Jahre vor der Veröffentlichung von Baldes Lyrica, bei Nicolaus Henricus d. J. ${ }^{19}$ in München erschienen war. In ihm erscheint Wigos Schrift unter der Überschrift Magni P. (= Patris) Wigonis, seu Guigonis, gravissimae considerationes de tranquillitate animi (pp. 134-291), also unter derselben Überschrift wie bei Balde, nur dass als alternativer Name des Verfassers der Name Guigo hinzugefügt ist. Dies ist der Name, unter dem der Verfasser üblicherweise zitiert wird. Gemeint ist Guigo de Castro (französisch Guigues du Chastel), der von 1109 bis zu seinem Tod 1136 (fünfter) Prior der Großen Kartause war. Den Text der ihm zugeschriebenen Schrift De tranquillitate animi hat der unbekannte Herausgeber des Sammelbandes, wie er in seinem Vorwort vermerkt, aus der „Magna bibliotheca patrum" übernommen. ${ }^{20}$ Dort ist er unter der Überschrift Venerabilis Patris Guigonis Carthusiani Meditationes aliquot pientissimae abgedruckt (tom. 12 pars 2 pp. 327-338). Bei Wigos considerationes de tranquillitate animi handelt es sich mithin um Guigos $\mathrm{Me}$ ditationes in der Form, in der sie damals und bis ins 20. Jh. hinein gelesen wurden. ${ }^{21}$ Wie wir heute wissen, war dies eine stark verkürzte und durcheinandergewirbelte Fassung der ursprünglichen Schrift. Die vollständige Fassung hat erst 1935 André Wilmart in einer Textausgabe zugänglich gemacht. ${ }^{22}$

In seinem Vorwort zu dem Sammelband mit den drei Schriften des Eucherius, des Martinus und Wigos wendet sich der unbekannte Verfasser mit Entschiedenheit gegen die stoische Apathie-Lehre, die besagt, dass man sich, wolle man zum inneren Frieden gelangen, gegen alle Triebe und Affekte immun machen müsse. Als Argument dafür, zu was für absurden Konsequenzen diese Lehre führe, nennt er folgendes Beispiel: ${ }^{23}$

„Wie schändlich ist jenes Hirngespinst des Phrygers, dass man den Verlust von Frau und Kindern nicht anders hinnehmen müsse, als wenn einem Kind die Meeresschnecken, an denen es sich erfreute, zertreten worden wären.“

Dass sich diese Kritik auf Ench. 7 bezieht, ist offenkundig. ${ }^{24}$ Offenkundig ist freilich auch, dass das, was bei Epiktet steht, in grob verfälschter Form erscheint. In zwei der drei christlichen Adaptionen des Encheiridions ist der originale Text Epiktets so gut wie

19 Vgl. zu ihm Benzing, Reske 2015, 679-680.

20 Margarin de la Bigne (Hg.): Magna bibliotheca veterum patrum et antiquorum scriptorum ecclesiasticorum, 14 Bände, Köln 1618-1622.

${ }_{21}$ Der Titel De tranquillitate animi findet sich, soweit ich sehe, nur in der Münchener Ausgabe von 1639 und in deren Nachdruck Brüssel 1671. Den Nachdruck Augsburg 1812, den Laporte 1983, 73 erwähnt, konnte ich nicht ausfindig machen. Auch den beiden anderen in dem Band enthaltenen Schriften hat der Herausgeber eigene, vom Üblichen abweichende Titel gegeben: Eucherius Lugdunensis, De vera sapientia = Epistola paraenetica ad Valerianum cognatum, de contemptu mundi et saecularis philosophiae (PL 50, 711-726), und Martinus Bracarensis, De vera virtute = Formula vitae honestae (PL 72, 23-28).

22 Wilmart 1936. Vgl. dazu Laporte 1983, 72-76 und Mursell 1995, $20-24$.

23 Wigo 1639, Vorwort: illud Phrygis quam contumeliosum figmentum est, iacturam uxoris et liberorum non aliter accipiendam, quam si infanti protritae fuerint quibus delectabatur cochleolae.

${ }^{24}$ Wie Balde in seiner Ode nennt übrigens auch der Autor dieses Vorwortes den Namen Epiktets nicht, sondern spricht von ihm als „dem Phryger“. 
unverändert übernommen, in einer, der Paraphrasis Christiana (Boter 1999, 373), ist er etwas ,entschärft' („eine Frau und ein Kind“ hat der Autor durch „Brüder oder Freunde oder Verwandte oder ein Haus" ersetzt). Die Verfasser dieser Adaptionen sahen also keinen Anlass, Anstoß an dem zu nehmen, was bei Epiktet zu lesen war, und in der Tat lässt es sich durchaus mit Worten Jesu vereinbaren. ${ }^{25}$ Empören kann sich der Verfasser des Vorwortes zu dem Sammelband von 1639 nur deshalb, weil er Epiktet etwas in den Mund legt, was dieser gar nicht gesagt hat. Man kann wohl ausschließen, dass er Epiktets Worte, um sie als Zielscheibe benutzen zu können, wissentlich in grober Weise verfälscht hat. Offenbar handelt es sich bei dem, was er Epiktet als Aussage zuschreibt, um vage Reminiszenzen an etwas, was er entweder selbst einmal bei Epiktet gelesen hatte oder, wahrscheinlicher noch, was er als grob verfälschende Epiktet-Reminiszenz bei einem anderen gefunden hatte.

Beim Zustandekommen der Aussage, die der Anonymus Epiktet zuschreibt, könnte im Übrigen eine andere vage Epiktet-Reminiszenz mitgespielt haben. ${ }^{26}$ Am Beginn von Diss. 4, 7 wirft Epiktet die Frage auf: „Was macht einen Tyrannen zum Gegenstand der Furcht?" Er konstatiert, dass es Menschen gibt, die weder unbedingt leben noch unbedingt sterben wollen, und die deshalb vor einem Tyrannen keine Angst haben. Und er fährt fort (Diss. 4, 7, 5):

„Wenn sich nun jemand auch seinem Besitz und seinen Kindern und seiner Frau gegenüber genauso verhält wie dieser seinem Körper gegenüber und wenn er sich, kurz gesagt, aufgrund einer Form von Wahnsinn und Verzweiflung in einer solchen Verfassung befindet, dass es ihn nicht im Mindesten interessiert, ob er diese Dinge besitzt oder nicht, wenn er vielmehr wie die Kinder, die mit Tonscherben spielen, ${ }^{27}$ zwar in Bezug auf das Spiel miteinander streiten, sich aber nicht um die Tonscherben kümmern - wenn so auch dieser Mensch die materiellen Dinge für nichts achtet, sondern nur das Spiel und die Beschäftigung mit ihnen schätzt, was für ein Tyrann, was für Leibwächter oder was für Schwerter können diesem Mann noch Furcht einflößen?“

Auch in diesem Text kommen Kinder und Frau als etwas vor, was jemandem gleichgültig ist. Von hier könnte die Verbindung von Gleichgültigkeit gegenüber Kindern und Frau einerseits und Kinderspielsachen andererseits stammen, die der Anonymus in seinem Vorwort Epiktet zuschreibt. Zwei vage Reminiszenzen an Dinge, die bei Epiktet zu lesen waren, könnten hier zusammengeflossen sein. Mag dies so sein oder auch nicht, deutlich wird aufgrund dessen, was im Vorangehenden aufgezeigt wurde, auf jeden Fall, wie es dazu kommen konnte, dass die von Epiktet Ench. 7 erwähnte „Meeresschnecke“ sich aus dem Kontext löste, sich gleichsam verselbständigte und zu jener Chiffre für die Verkehrtheit und Nutzlosigkeit der Philosophie Epiktets wurde, als die Balde sie in seiner Ode benutzt.

Epiktets „von schwarzem Licht qualmende Lampe“ (V. 10) erwähnt Balde auch in einer der beiden Oden, in denen er begründet, warum sich seine anfängliche Sympathie für die stoische Philosophie ins Gegenteil verkehrt hat, der Ode 3, 16. Rückblickend schreibt er hier über die Zeit, zu der er sich zur stoischen Philosophie hingezogen fühlte: ${ }^{28}$ „und schon freute ich mich, von Epiktets Leuchte im Gesicht geschwärzt, vom Ruß gefärbt zu

25 Vgl. Mt. 10, 37 und 19, 29; Luk. 9, 59-62; ferner Paulus 1. Kor. 7, 29.

26 Diese Verbindung hat Neubig 1828, 223 in einer seiner Anmerkungen zur Ode 3, 39 hergestellt.

$27 \mathrm{Zu}$ den Tonscherbenspielen vgl. Grasberger 1864-1881, 1, 57-60.

${ }^{28}$ Lyr. 3, 16, 25-26: Iamque ab Epicteti factus niger ora lucerna / Gaudebam fuligine tingi. 
werden“. Epiktet erzählt in den Dissertationes, dass er, als ihm eines Abends seine eiserne Lampe gestohlen worden war, dies mit großer Gelassenheit hingenommen und zu sich gesagt habe, dass er sich ja am nächsten Tag eine einfachere tönerne kaufen könne $(1,18$, 15). Und an einer anderen Stelle $(1,29,21)$ merkt er an, dass dieser Vorfall für den Dieb schlimmer gewesen sei als für ihn, denn dieser habe durch den Diebstahl seiner seelischen Integrität Schaden zugefügt. Doch das ist nicht das, was Balde im Blick hatte, als er Epiktets Lampe in den Oden 3, 16 und 3, 39 erwähnte. Was er im Blick hatte, ergibt sich aus den Erwähnungen der tönernen Lampe in zwei anderen seiner Dichtungen. Im Agathyrsus heißt es von Epiktet: ${ }^{29}$ „Mager war der, ... dessen tönerne Leuchte .... das Hausgerät des Sklaven, für strahlende Smaragde verkauft wurde." und im Torvitatis encomium: ${ }^{30}$ „Epiktets Lampe, die für 300 Münzen verkauft wurde, wird, wiewohl er seinen Nachttopf in seiner Bettdecke reinigte, ${ }^{31}$ bis heute aufbewahrt; und sie versorgt seinen strahlenden Ruhm mit Öl. Sie wird so in alle Ewigkeit brennen, dass sie nur dann auszulöschen sein wird, wenn auch die Gestirne dahinschwinden." Quelle für den in den beiden Texten erwähnten Verkauf der Lampe Epiktets ist eine Geschichte, die bei Lukian überliefert ist (Adv. indoct. 13; Übers. Chr. M. Wieland):

${ }^{29}$ Agathyrsus XLVIII (opp. poet. omn. 7, 265): Macer fuit, ... / Cuius lucerna fictilis, / Servi supellex, vaeniit / Fulgentibus smaragdis.

30 Torvitatis encomium VI (opp. poet. omn. 3, 362): ... ter centum vendita nummis / Lampas Epicteti, quanquam in lodice matellam / Purgantis, servatur adhuc; oleumque ministrat / Lucenti famae; quae sic ardebit in aevum, / Ut nisi cum lapsis non exstinguenda sit astris.

$31 \mathrm{Zu}$ der einigermaßen seltsam anmutenden Behauptung, Epiktet habe „seinen Nachttopf in seiner Bettdecke gereinigt“: Bei dem Torvitatis encomium handelt es sich um ein sog. Paradox-Encomium, d. h. ein Encomium, das die Dinge gleichsam auf den Kopf stellt, indem es etwas rühmt, was gemeinhin negativ konnotiert ist, in diesem Fall die Eigenschaft des Wilden, Grimmigen, Finsteren, auch Ungepflegten. Zum Ruhme der torvitas nennt Balde in der Schrift neben vielem anderen zahlreiche Philosophen, die trotz oder gerade wegen ihrer torvitas großes Ansehen genossen hätten und weiterhin genössen. Bei der Charakterisierung dieser Philosophen und der Beschreibung der Eigenschaften, die ihre torvitas beweisen sollen, lässt Balde seiner Fantasie freien Lauf und streut auch skurrile Erfindungen ein (vgl. etwa die Charakterisierungen des Stoikers Chrysipp und des Akademikers Speusipp, Torv. enc. III und VI, opp. poet. omn. 3, 360 und 362). Eine solche Erfindung ist offenkundig auch die Behauptung, Epiktet habe „seinen Nachttopf in seiner Bettdecke gereinigt". Natürlich stellt sich die Frage, was Balde veranlasst haben mag, Epiktet gerade

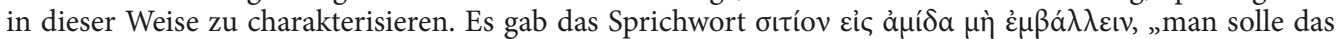
Getreide nicht in einen Nachttopf werfen" (Plut. De lib. ed. 17, 12F; vgl. Hor. Ep. 1, 2, 54), was dem Sinne nach unserem Sprichwort entspricht, „man solle die Perlen nicht vor die Säue werfen“. Erasmus hat dieses Sprichwort in seine Adagia aufgenommen $(1,1,2,9)$ und mit einem bei Gellius überlieferten Ausspruch Epiktets verbunden (Gell. NA 17, 19, 1-4=Epiktet fr. X Schenkl). Als Epiktet, so heißt es bei Gellius, gesehen habe, wie ein moralisch durch und durch verdorbener Mensch sich der Philosophie zugewandt und Fragen der Physik, der Dialektik und anderer Disziplinen zu erforschen begonnen habe, habe er ihn mit folgenden Worten angefahren: „Mensch, wo deponierst du diese Dinge? Sieh zu, ob das Gefäß gereinigt ist. Deponierst du sie nämlich in deiner vorgefassten Meinung (oínбıৎ), sind sie ruiniert; wenn sie verfaulen, werden sie zu Urin oder Essig oder womöglich zu etwas noch Üblerem als diesen.“ Möglicherweise waren das zitierte Sprichwort und/oder der von Gellius zitierte Ausspruch Epiktets Balde bekannt und gaben ihm den Anstoß zu seiner Behauptung, Epiktet habe „seinen Nachttopf in seiner Bettdecke gereinigt“. Auf jeden Fall galt der Nachttopf als Inbegriff des Schmutzigen und Unappetitlichen, weshalb es denn auch als etwas zutiefst Erniedrigendes angesehen wurde, jemandem einen Nachttopf reichen zu müssen (vgl. Epict. Diss. 1, 2, 8-11). Worum es Balde geht, ist, dass es Epiktets ewig währendem Ruhm in keiner Weise geschadet habe, dass er nichts dabei gefunden habe, etwas so Ordinäres zu tun, wie „seinen Nachttopf in seiner Bettdecke zu reinigen“. - Nebenbei bemerkt: Balde sagt nicht, Epiktet habe seinen Nachttopf „mit seiner Bettdecke“ (lodice) gereinigt, was metrisch auch möglich gewesen wäre, sondern ,in seiner Bettdecke“ (in lodice). Was genau damit gemeint ist, ist mir unklar. 
„In unseren heutigen Tagen hat jemand gelebt und lebt vielleicht noch, der für die Lampe des Epiktetos, wiewohl sie nur von Ton war, dreitausend Drachmen bezahlte. Vermutlich muss der Mann geglaubt haben, er brauche nur bei dieser Lampe zu lesen, so werde ihm die Weisheit jenes allgemein bewunderten Greises im Schlafe kommen und er also mit größter Bequemlichkeit ein zweiter Epiktetos werden können."

Da Lukian nur etwa zwei Generationen jünger war als Epiktet, muss diese Geschichte schon bald nach Epiktets Tod aufgekommen sein. Als die Schriften Lukians und Epiktets nach der Eroberung Konstantinopels 1453 in den Westen gelangten und dort erstmals gedruckt und übersetzt wurden, ${ }^{32}$ wurde auch diese Geschichte bekannt. Poliziano erwähnt sie in dem Begleitbrief, den er seiner lateinischen Übersetzung des Encheiridions beifügte, als er sie 1479 Lorenzo de’ Medici übersandte: ${ }^{33}$ „Die Bewunderung für sein (sc. Epiktets) Leben war bei allen Menschen so groß, dass der Syrer Lukian überliefert, Epiktets tönerne Lampe sei wegen seines Ansehens für dreitausend Drachmen verkauft worden.“ Und er fügt mit Bezug auf seine Übersetzung des Encheiridions hinzu: „Wir senden dir, Lorenzo, jetzt nicht die Lampe Epiktets, sondern das Abbild seines Geistes, das viel mehr Licht spendet." Bis in die Gegenwart war die Kenntnis dieser Geschichte weit verbreitet. ${ }^{34}$ Angesichts der Tatsache, dass Balde in zwei seiner Dichtungen ausdrücklich auf sie anspielt, ist es sehr wahrscheinlich, dass er sie auch in den beiden Oden 3, 16 und 3, 39 im Blick hatte, wenn er Epiktets Lampe dort als Symbol für die Philosophie Epiktets benutzte. Dass Balde sich eingehender mit Epiktets Schriften und seiner Philosophie beschäftigt hätte, lässt sich aus der Erwähnung der Lampe in den Oden 3, 16 und 3, 39 nicht herleiten.

Bleibt die Frage, was Balde veranlasste, gerade Epiktet und speziell sein Encheiridion als Exponenten der stoischen Philosophie Wigos De tranquillitate animi (= Guigos Meditationes) gegenüberzustellen. Den Anstoß dazu, Epiktet als Antipoden zu wählen, gab, wie mir scheint, das Vorwort zu dem Sammelband von 1639, in dem der Autor die stoische Philosophie im Allgemeinen und Epiktet im Besonderen ebenso scharf kritisiert wie Balde in der Ode 3, 39. Wie im Vorangehenden gezeigt wurde, ist Baldes Verweis auf die „Meeresschnecken“ Epiktets als Beispiel für die Nutzlosigkeit seiner Philosophie nur verständlich, wenn man den Zusammenhang kennt, in dem diese „Meeresschnecken“ in dem Vorwort erwähnt werden. Allem Anschein nach hat Balde sie als Beispiel von dort übernommen. Wie gleichfalls gezeigt wurde, ist allerdings weder dem Autor des Vorwortes noch Balde bewusst, dass diese „Meeresschnecken“ auf Epiktets Encheiridion zurückgehen. Hätten sie es gewusst, hätten sie das Beispiel nicht in der Weise benutzten können, in der sie dies tun. Der Autor des Vorwortes erwähnt das Encheiridion nicht und es ist durchaus fraglich, ob er es überhaupt kannte. Wie gut Balde das Encheiridion kannte, sei dahingestellt; so gut, dass er gewusst hätte, in welcher Weise Epiktet in ihm das Beispiel der „Meeresschnecke“ gebraucht, kannte er es jedenfalls nicht. Dass er dennoch gerade das Encheiridion zum Angriffsziel macht, erklärt sich wohl in der Weise, dass diese Schrift zu seiner Zeit

32 Editio princeps der Schriften Lukians 1496, des Encheiridions Epiktets 1529, seiner Dissertationes 1535; zu den frühesten Übersetzung des Encheiridions vgl. Döring 2010, 320. 329-330.

33 Politianus 1519, tomus 2 p. 30v : Tanta ... apud omneis homines eius vitae admiratio extitit, ut Lucianus Syrus Epicteti lucernam fictilem tribus drachmarum milibus ob eius auctoritatem venisse tradat. Nos ad te nunc non lucernam Epicteti, sed eius imaginem animi, quae multo plus lucis habeat, Laurenti, mittimus.

${ }^{34}$ Dies im Einzelnen auszuführen, würde eine eigene Abhandlung erfordern. Angemerkt sei nur, dass auch Jeremias Drexel die Geschichte erwähnt (Heliotropium II 3, 2 [Drexel 1627, 106]): quae lucerna a morte eius mille drachmis hoc est centum philippeis vaeniit in memoriam et honorem tanti viri. 
und nicht zum Wenigsten unter seinen Mitbrüdern, den Jesuiten, hohes Ansehen genoss. Epiktets Encheiridion muss als Sündenbock für die Philosophie der Stoiker im Allgemeinen herhalten.

\section{Literatur}

Amigues S. (Éd.) Théophraste: Recherches sur les plantes. Texte établi et traduit. T. 4, Paris, Les Belles Lettres, 2003.

Arend S. „Nec Lapis esse volo“ — „Und kein Stein will ich sein“. Zum antistoischen Affekt in der Lyrik Jacob Baldes, in: Burkard, Thorsten u. a. (Hg.): Jacob Balde im kulturellen Kontext seiner Epoche. Zur 400. Wiederkehr seines Geburtstages. Regensburg, Schnell \& Steiner 2006, 153-165.

Benzing J., Reske Chr. Die Buchdrucker des 16. und 17. Jahrhunderts im deutschen Sprachgebiet. Wiesbaden, Harrassowitz ${ }^{2} 2015$.

Boter G. (Ed.) The Encheiridion of Epictetus and its three Christian adaptations. Transmission and critical editions. Leiden - Boston, Brill, 1999.

Döring K. Zur Rezeption von Epiktets Encheiridion, in: Ders. Kleine Schriften zur antiken Philosophie und ihrer Nachwirkung, Stuttgart, Steiner, 2010, 319-343.

Drexel H. [Hieremias Drexelius] Heliotropium seu conformatio humanae voluntatis cum divina, München, Cornelius Leysserius, 1627.

Grasberger L. Erziehung und Unterricht im klassischen Altertum. Nach den Quellen dargestellt; in 3 Teilen. Würzburg, Stahel, 1864-1881 [ND: Aalen, Scientia, 1971].

Hadot I. (Éd.) Simplicius: Commentaire sur le Manuel d'Épictète. Introduction et édition critique du texte grec. Leiden - New York, Brill, 1996.

Hünemörder Chr. Meerzwiebel, in: Der Neue Pauly 7, 1999, 1130-1131.

Laporte M. (Éd.) Guigues Ier, prieur de Chartreuse. Les Méditations (Recueil de Pensées). Introduction, texte critique, traduction et notes par un Chartreux [Maurice Laporte]. Paris, Éditions du Cerf, 1983.

Leumann M. Kleine Schriften, Zürich - Stutgart, Artemis, 1959.

Mueller P. (Hrsg.) Balde, Jacob: Carmina lyrica. Recognovit annotationibusque illustravit P. Benno Mueller. Regensburg, Olms, ${ }^{2} 1884$ [ND: Hildesheim, Olms, 1977].

Mursell A. Guigo I, Prior of the Charterhouse: The meditations, transl. with an introduction by A. Gordon Mursell, Kalamazoo, Mich., Cistercian Publications, 1995.

Neubig J. Bavaria’s Musen in Joh. Jak. Balde’s Oden, aus dem Latein ins das Vermaß der Urschrift übersetzt. München, Gielsche Buchhandlung, 1828.

Pörnbacher K. Jeremias Drexel. Leben und Werk eines Barockpredigers, München, Seitz, 1965.

Politianus Angelus. Omnia opera. Paris, Josse Bade, 1519.

Spanneut M. (Êd.) Commentaire sur la Paraphrase chrétienne du Manuel d'Épictète. Introduction, texte (partiellement) inédit, apparat critique, traduction, notes et index par Michel Spanneut, Paris, Le Cerf, 2007.

Tan K., Strid A. Wild flowers of Greece. Colour plates by Niki A. Goulandris. Kifissia, Greece, The Goulandris Natural History Museum, 2009.

Vollenweider S. Lebenskunst als Gottesdienst. Epiktets Theologie und ihr Verhältnis zum Neuen Testament, in: Ders. (Hrsg.) Epiktet: Was ist wahre Freiheit. Diatribe IV 1. Tübingen, Mohr Siebeck, 2013, 119-162.

Wilmart A. (Éd.) Le recueil des pensées du B. (= Bienheureux) Guigue. Édition complète, accompagnée de tables et d'une traduction. Paris, Vrin, 1936.

Received: 09.02.2018

Final version received: 21.04 .2018

\section{Якоб Бальде об Эпиктете и его «Руководстве»}

\section{Клаус Дёринг}

Среди различных отражений «Энхиридиона» и многих похвал Эпиктету в литературе раннего Нового времени обнаруживаются редкие, а потому нуждающиеся в объяснении вкрапления критики. Так, Якоб Бальде, прославленный новолатинский поэт, принадлежавший к ордену иезуитов, в одной из своих «Од» $(3,39)$ предполагает во- 
оружиться не «кинжалом раба» (игра слов: «энхейридион» означает как «руководство», так и «кинжал»), а мечом, «который дал Павел» (словом Божьим: Еф. 6 : 17), и советует «читать Виго», оставив стоиков с их «привычным бормотанием о ракушках курносого фригийца, тусклой чадящей лампаде» и т.д. Аллюзия на известное место «Руководства» (Ench. 7) очевидна, но вместе с тем и странна: ведь этот текст никак не противоречит христианской мысли. Философ советует бросить - как ракушки, которые собирает пассажир, сошедший на берег во время недолгой остановки, - своих детей и жену, когда капитан корабля, - то есть бог - просигналит отплытие. Пафос созвучного евангельского призыва, конечно, иной, но приблизить стоическую проповедь к христианской удавалось и сквозь более непроницаемые заслоны, тогда как именно здесь адаптация напрашивается (и осуществлялась другими авторами). Бальде сам подсказал объяснение своего неудачного выбора: «Виго» - это трактат De tranquillitate animi (полный титул: De vera sapientia, virtute et tranquillitate animi enchiridion DD. Eucherii Lugdunensis, Martini Bracarensis, et Magni Wigonis Antistitum) 1639 года: отсюда поэт почерпнул свои сведения об Эпиктете. Место о ракушках критикуется «Вигоном» (Гвиго де Кастро), однако - в переиначенном виде: «утрату жены и детей», - думал якобы «фригиец» (!), - «нужно воспринимать так, словно бы у ребенка раздавили, наступив на них, ракушки, с которыми он забавлялся». Лишь в таком, искаженном виде слова Эпиктета могли вызвать негодование христианина. Впрочем, анонимный автор трактата исказил античный источник не намеренно: переделка явилась результатом неосознанной компиляции двух пассажей (cp. Diss. 4, 7, 5).

Ключевые слова: Эпиктет, Бальде, Гвиго де Кастро, Энхиридион. 